\title{
Precision Measurement of the Mass and Lifetime of the $\Xi_{b}^{-}$Baryon
}

\author{
R. Aaij et al. \\ (LHCb Collaboration) \\ (Received 30 September 2014; published 9 December 2014)
}

\begin{abstract}
We report on measurements of the mass and lifetime of the $\Xi_{b}^{-}$baryon using about $1800 \Xi_{b}^{-}$decays reconstructed in a proton-proton collision data set corresponding to an integrated luminosity of $3.0 \mathrm{fb}^{-1}$ collected by the LHCb experiment. The decays are reconstructed in the $\Xi_{b}^{-} \rightarrow \Xi_{c}^{0} \pi^{-}, \Xi_{c}^{0} \rightarrow p K^{-} K^{-} \pi^{+}$ channel and the mass and lifetime are measured using the $\Lambda_{b}^{0} \rightarrow \Lambda_{c}^{+} \pi^{-}$mode as a reference. We measure $M\left(\Xi_{b}^{-}\right)-M\left(\Lambda_{b}^{0}\right)=178.36 \pm 0.46 \pm 0.16 \mathrm{MeV} / c^{2},\left(\tau_{\Xi_{b}^{-}} / \tau_{\Lambda_{b}^{0}}\right)=1.089 \pm 0.026 \pm 0.011$, where the uncertainties are statistical and systematic, respectively. These results lead to a factor of 2 better precision on the $\Xi_{b}^{-}$mass and lifetime compared to previous best measurements, and are consistent with theoretical expectations.
\end{abstract}

DOI: 10.1103/PhysRevLett.113.242002

PACS numbers: 14.20.Mr, 13.30.Eg

Over the last two decades, beauty mesons have been studied in detail. Various theoretical approaches allow one to relate measured decay rates to standard model parameters. One of the most predictive tools is the heavy quark expansion (HQE) [1-8], which describes the decay rates of beauty hadrons through an expansion in powers of $\Lambda_{\mathrm{QCD}} / m_{b}$, where $\Lambda_{\mathrm{QCD}}$ is the energy scale at which the strong-interaction coupling becomes large, and $m_{b}$ is the $b$-quark mass. In addition to the total $b$-hadron decay widths, $\mathrm{HQE}$ can be used to calculate $b$-hadron parameters required for the measurement of coupling strengths between quarks in charged-current interactions, which in turn provides constraints on physics beyond the standard model.

A stringent test of $\mathrm{HQE}$ is to confront its predictions for lifetimes, i.e., the inverse of the corresponding decay widths, with precision measurements. The lifetimes of the $B^{0}$ and $B^{+}$mesons are measured to a precision of about $0.5 \%$ [9], the $B_{s}^{0}$ meson to $1 \%[9,10]$, and the $\Lambda_{b}^{0}$ baryon to $0.7 \%$ [9], and their values are in agreement with HQE predictions [11].

Another interesting test is to compare the measured lifetime ratio $\tau\left(\Xi_{b}^{-}\right) / \tau\left(\Xi_{b}^{0}\right)$ to $\mathrm{HQE}$ predictions. Since penguin contraction terms cancel in this ratio [12], a more precise prediction is possible compared to $\tau\left(\Lambda_{b}^{0}\right) / \tau\left(B^{0}\right)$. One prediction leads to $\tau\left(\Xi_{b}^{-}\right) / \tau\left(\Xi_{b}^{0}\right)=1.05 \pm 0.07$ [12], where the dominant uncertainties are related to matrix elements that are calculable using lattice quantum chromodynamics (QCD) [13]. A phenomenological analysis of the relevant matrix elements using charm baryon lifetimes leads to a prediction of $1 / \tau\left(\Lambda_{b}^{0}\right)-1 / \tau\left(\Xi_{b}^{-}\right)=0.11 \pm$ $0.03 \mathrm{ps}^{-1}$ [14], or $\tau\left(\Xi_{b}^{-}\right) / \tau\left(\Lambda_{b}^{0}\right)=1.19_{-0.06}^{+0.07}$. Recently,

* Full author list given at the end of the article.

Published by the American Physical Society under the terms of the Creative Commons Attribution 3.0 License. Further distribution of this work must maintain attribution to the author(s) and the published articles title, journal citation, and DOI. the first measurement of the lifetime ratio $\tau\left(\Xi_{b}^{0}\right) / \tau\left(\Lambda_{b}^{0}\right)$ was made, yielding $\tau\left(\Xi_{b}^{0}\right) / \tau\left(\Lambda_{b}^{0}\right)=1.006 \pm 0.018 \pm 0.010$ [15]. Previous $\Xi_{b}^{-}$lifetime measurements, which used $\Xi_{b}^{-} \rightarrow J / \psi \Xi^{-}$decays, led to values of $1.55_{-0.09}^{+0.10} \pm$ 0.03 ps [16] and $1.32 \pm 0.14 \pm 0.02$ ps [17]. The weighted average of these two results, along with the recent $\Xi_{b}^{0}$ lifetime measurement [15], yields $\tau\left(\Xi_{b}^{-}\right) / \tau\left(\Xi_{b}^{0}\right)=$ $1.00 \pm 0.06$. Improved experimental and theoretical precision of the $\Xi_{b}^{-}$lifetime will allow for a more stringent test of the HQE prediction.

Measurements of $b$-baryon masses and isospin splittings provide information on the interquark potential. A number of QCD-inspired models predict the $\Xi_{b}^{0}$ and $\Xi_{b}^{-}$masses, or their average, which range from approximately 5780 to $5900 \mathrm{MeV} / \mathrm{c}^{2}$ [18-27]. More accurate predictions exist for the $\Xi_{b}^{-}-\Xi_{b}^{0}$ mass splitting, estimated to be $6.24 \pm 0.21 \mathrm{MeV} / c^{2}$ or $6.4 \pm 1.6 \mathrm{MeV} / c^{2}$ when extrapolating from the measured isospin splitting $M\left(\Xi^{-}\right)-M\left(\Xi^{0}\right)$ or $M\left(\Xi_{c}^{0}\right)-M\left(\Xi_{c}^{+}\right)$, respectively [22]. The $\Xi_{b}^{-}$mass is currently known to a precision of $1.0 \mathrm{MeV} / c^{2}$ [28], which is a factor of 3 less precise than that of the $\Xi_{b}^{0}$ baryon [15].

In this Letter, we report improved measurements of the mass and lifetime of the $\Xi_{b}^{-}$baryon using about 1800 $\Xi_{b}^{-} \rightarrow \Xi_{c}^{0} \pi^{-}, \Xi_{c}^{0} \rightarrow p K^{-} K^{-} \pi^{+}$signal decays. The measurements are normalized using the $\Lambda_{b}^{0} \rightarrow \Lambda_{c}^{+} \pi^{-}, \Lambda_{c}^{+} \rightarrow$ $p K^{-} \pi^{+}$decay as a reference. Charge conjugate processes are implied throughout.

The measurements use proton-proton ( $p p$ ) collision data samples, collected by the LHCb experiment, corresponding to an integrated luminosity of $3.0 \mathrm{fb}^{-1}$, of which $1.0 \mathrm{fb}^{-1}$ was recorded at a center-of-mass energy of $7 \mathrm{TeV}$ and $2.0 \mathrm{fb}^{-1}$ at $8 \mathrm{TeV}$. The LHCb detector [29] is a single-arm forward spectrometer covering the pseudorapidity range $2<\eta<5$, designed for the study of particles containing $b$ or $c$ quarks. The detector includes a high-precision tracking system, which provides a momentum measurement with 
precision of about $0.5 \%$ from $2-100 \mathrm{GeV} / c$ and impact parameter resolution of $20 \mu \mathrm{m}$ for particles with large transverse momentum $\left(p_{T}\right)$. The polarity of the dipole magnet is reversed periodically throughout data taking to reduce asymmetries in the detection of charged particles. Ring-imaging Cherenkov detectors [30] are used to distinguish charged hadrons. Photon, electron, and hadron candidates are identified using a calorimeter system, followed by detectors to identify muons [31].

The trigger [32] consists of a hardware stage, based on information from the calorimeter and muon systems, followed by a software stage, which applies a full event reconstruction [32,33]. About 57\% of the selected $X_{b}$ events are triggered at the hardware level by one or more of the $X_{b}$ final-state particles. [Throughout, we use $X_{b}\left(X_{c}\right)$ to refer to either a $\Xi_{b}^{-}\left(\Xi_{c}^{0}\right)$ or $\Lambda_{b}^{0}\left(\Lambda_{c}^{+}\right)$baryon.] The remaining $43 \%$ are triggered only on other activity in the event. We refer to these two classes of events as triggered on signal (TOS) and triggered independently of signal (TIS). The software trigger requires a two-, three-, or fourtrack secondary vertex with a large scalar $p_{T}$ sum of the particles and a significant displacement from the primary $p p$ interaction vertices (PVs). At least one particle should have $p_{T}>1.7 \mathrm{GeV} / c$ and be inconsistent with coming from any of the PVs. The signal candidates are required to pass a multivariate software trigger selection algorithm [33].

Proton-proton collisions are simulated using PYTHIA [34] with a specific LHCb configuration [35]. Decays of hadronic particles are described by EvTGEN [36], in which final-state radiation is generated using Рнотоs [37]. The interaction of the generated particles with the detector and its response are implemented using the GEANT4 toolkit [38] as described in Ref. [39]. The $X_{c}$ final states are modeled using a combination of resonant and nonresonant contributions to reproduce the substructures seen in data.

Signal $\Xi_{b}^{-}\left(\Lambda_{b}^{0}\right)$ candidates are formed by combining in a kinematic fit a $\Xi_{c}^{0} \rightarrow p K^{-} K^{-} \pi^{+}\left(\Lambda_{c}^{+} \rightarrow p K^{-} \pi^{+}\right)$candidate with a $\pi^{-}$candidate (referred to as the bachelor). The $X_{b}$ candidate is included in the fit to each PV and is then associated with the one for which the $\chi^{2}$ increases by the smallest amount. The kinematic fit exploits PV, $X_{b}$, and $X_{c}$ decay-vertex constraints to improve the mass resolution. The $X_{c}$ decay products are each required to have $p_{T}>100 \mathrm{MeV} / c$, and the bachelor pion is required to have $p_{T}>500 \mathrm{MeV} / c$. All final-state particles from the signal candidate are required to have trajectories that are significantly displaced from the PV and to pass particle identification (PID) requirements. The $K^{-}$and $\pi^{+}$PID efficiencies are determined from $D^{*+} \rightarrow D^{0} \pi^{+}, D^{0} \rightarrow$ $K^{-} \pi^{+}$calibration samples, whereas the proton PID efficiency is determined from simulation. The PID efficiencies are reweighted to account for different momentum spectra and track occupancies between the calibration and signal samples. The efficiencies of the PID requirements on the
$\Xi_{c}^{0}$ and $\Lambda_{c}^{+}$final states are $80 \%$ and $86 \%$, respectively. Mass vetoes are used to suppress cross feeds from misidentified $D_{(s)}^{+} \rightarrow K^{+} K^{-} \pi^{+}, \quad D^{*+} \rightarrow D^{0}\left(K^{+} K^{-}\right) \pi^{+}, \quad$ and $D^{+} \rightarrow$ $K^{-} \pi^{+} \pi^{+}$decays faking $\Lambda_{c}^{+} \rightarrow p K^{-} \pi^{+}$decays, as in Ref. [15]. The difference between the $\Xi_{c}^{0}\left(\Lambda_{c}^{+}\right)$candidate mass and the known value [9] is required to be less than $14 \mathrm{MeV} / c^{2}\left(20 \mathrm{MeV} / c^{2}\right)$, which is about 2.5 times the mass resolution.

To improve the signal-to-background ratio, we employ a boosted decision tree (BDT) discriminant $[40,41]$ built from the same variables used in Ref. [15]. To train the BDT, the kinematic distributions of the signal are modeled using simulated decays. The background is modeled using signal candidates with $X_{b}$ invariant mass greater than $300 \mathrm{MeV} / c^{2}$ above the signal peak mass. To increase the size of the background sample for the $\Xi_{b}^{-}$BDT training, we also include events in the $\Xi_{c}^{0}$ sideband regions, $20<\left|M\left(p K^{-} K^{-} \pi^{+}\right)-M\left(\Xi_{c}^{0}\right)\right|<50 \mathrm{MeV} / c^{2}$. The BDT requirement is chosen to minimize the expected $\Xi_{b}^{-}$relative yield uncertainty, corresponding to a selection efficiency of 97\% (50\%) for signal (combinatorial background). The fraction of events with multiple candidates is below $1 \%$ (mostly one extra candidate) over the full fit range in both the signal and normalization modes. All candidates are kept.

The invariant mass signal shapes are obtained from simulated $\Xi_{b}^{-} \rightarrow \Xi_{c}^{0} \pi^{-}$and $\Lambda_{b}^{0} \rightarrow \Lambda_{c}^{+} \pi^{-}$decays. They are each modeled by the sum of two Crystal Ball (CB) functions [42] with a common mean as

$$
\begin{aligned}
f_{\text {sig }}^{\Lambda_{b}^{0}}= & f_{\text {low }} \mathrm{CB}_{-}\left(m_{0}, \sigma_{-}, \alpha_{-}, n\right) \\
& +\left(1-f_{\text {low }}\right) \mathrm{CB}_{+}\left(m_{0}, \sigma_{+}, \alpha_{+}, n\right) \\
f_{\text {sig }}^{\Xi_{b}^{-}}= & f_{\text {low }} \mathrm{CB}_{-}\left(m_{0}^{\prime}, f_{\sigma} \sigma_{-}, f_{\alpha_{-}} \alpha_{-}, n\right) \\
& +\left(1-f_{\text {low }}\right) \mathrm{CB}_{+}\left(m_{0}^{\prime}, f_{\sigma} \sigma_{+}, f_{\alpha_{+}} \alpha_{+}, n\right) .
\end{aligned}
$$

The CB functions each include a Gaussian component to describe the core of the mass distribution, as well as powerlaw tails to describe the radiative tail below $\left(\mathrm{CB}_{-}\right)$and the non-Gaussian resolution above $\left(\mathrm{CB}_{+}\right)$the signal peak. The extent of these tails is governed by the width and tail parameters, $\sigma_{ \pm}$and $\alpha_{ \pm}$, respectively. The parameter $m_{0}$ is the fitted $\Lambda_{b}^{0}$ mass, and $m_{0}^{\prime} \equiv m_{0}+\delta M$ is the $\Xi_{b}^{-}$mass, written in terms of the fitted mass difference $\delta M$ between the two signals. The low-mass $\mathrm{CB}$ width $\sigma_{-}$is expressed in terms of the high-mass width using $\sigma_{-}=r_{\sigma} \sigma_{+}$. The parameters $f_{\sigma}$ and $f_{\alpha_{ \pm}}$allow for possible differences in the mass resolutions and tail parameters, respectively, between the signal and normalization modes. We fix the power $n=10$ and $f_{\text {low }}=0.5$ to minimize the number of correlated parameters in the signal shape. The parameters $r_{\sigma}, f_{\alpha_{+}}, f_{\alpha_{-}}$, and $f_{\sigma}$ are determined from simulated decays, and they are consistent with unity. These four parameters 

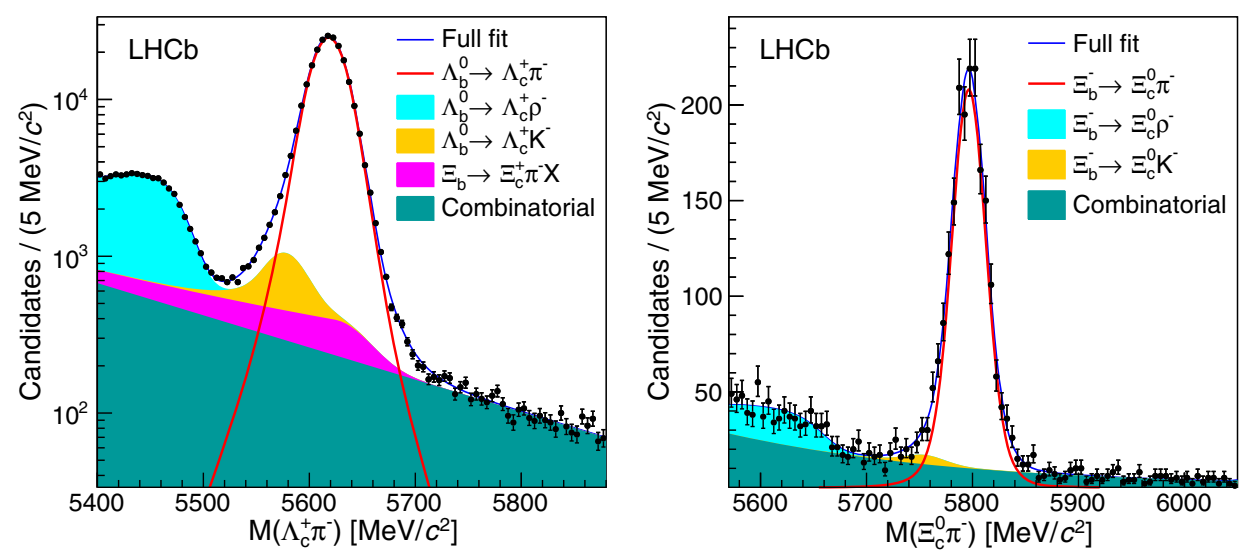

FIG. 1 (color online). Invariant mass spectrum, along with the fit projections, for (left) $\Lambda_{b}^{0} \rightarrow \Lambda_{c}^{+} \pi^{-}$and (right) $\Xi_{b}^{-} \rightarrow \Xi_{c}^{0} \pi^{-}$candidates.

are fixed in fits to the data to the values from simulation, while $\sigma_{+}, \alpha_{+}$, and $\alpha_{-}$are freely varied, along with $m_{0}$ and $\delta M$.

The invariant mass spectra also include partially reconstructed $b$-baryon background contributions, misidentified $K^{-}$in $X_{b} \rightarrow X_{c} K^{-}$decays, charmless backgrounds, as well as random track combinations, primarily from false $X_{c}$ candidates. The main source of partially reconstructed background is from $X_{b} \rightarrow X_{c} \rho^{-}$decays, where a $\pi^{0}$ from the $\rho^{-}$decay is not used to form the candidate. Its shape is obtained from simulated $\Lambda_{b}^{0} \rightarrow \Lambda_{c}^{+} \rho^{-}$decays, and is assumed to be the same for both the signal and normalization modes, apart from a shift in the overall mass spectrum. A contribution from $\Lambda_{b}^{0} \rightarrow \Sigma_{c}^{+} \pi^{-}, \Sigma_{c}^{+} \rightarrow \Lambda_{c}^{+} \pi^{0}$ decays is also expected to populate the $\Lambda_{c}^{+} \pi^{-}$mass spectrum, and its shape is taken to be the same to that of the $\Lambda_{b}^{0} \rightarrow \Lambda_{c}^{+} \rho^{-}$signal. An additional contribution from partially reconstructed $\Xi_{b}$ decays is found, through a study of the $\Lambda_{c}^{+}$sidebands, to populate the $\Lambda_{c}^{+} \pi^{-}$mass spectrum. This background is modeled through a fit to the $\Lambda_{b}^{0}$ candidate mass spectrum obtained using the lower and upper $\Lambda_{c}^{+}$mass sidebands. The shape of the background from misidentified $X_{b} \rightarrow X_{c} K^{-}$decays is taken from simulation. The misidentification rate of $3.1 \%$ is obtained from $D^{*+} \rightarrow D^{0} \pi^{+}$calibration samples, reweighted in $p_{T}$, $\eta$, and number of tracks to match the distributions observed in data. No peaking contributions from charmless backgrounds are observed when studying the $X_{b}$ mass spectra using the $X_{c}$ mass sidebands. The combinatorial background is modeled using an exponential function with a freely varying slope.

The $\Lambda_{c}^{+} \pi^{-}$and $\Xi_{c}^{0} \pi^{-}$mass spectra are fit simultaneously using a binned maximum likelihood fit. The results of the fit are shown in Fig. 1. A total of $1799 \pm 46 \Xi_{b}^{-} \rightarrow \Xi_{c}^{0} \pi^{-}$ and $(220.0 \pm 0.5) \times 10^{3} \Lambda_{b}^{0} \rightarrow \Lambda_{c}^{+} \pi^{-}$signal decays are observed. The mass difference is measured to be

$$
\delta M \equiv M\left(\Xi_{b}^{-}\right)-M\left(\Lambda_{b}^{0}\right)=178.36 \pm 0.46 \mathrm{MeV} / c^{2},
$$

where the uncertainty is statistical only.
The observed signals are also used to measure the $\Xi_{b}^{-}$ baryon lifetime relative to that of the $\Lambda_{b}^{0}$ baryon. We measure the efficiency-corrected yields in six bins of measured decay time, as given in Table I. The ratio of efficiency-corrected yields depends exponentially on decay time as $N_{\text {cor }}\left[\Xi_{b}^{-} \rightarrow \Xi_{c}^{0} \pi^{-}\right](t) / N_{\text {cor }}\left[\Lambda_{b}^{0} \rightarrow \Lambda_{c}^{+} \pi^{-}\right](t)=e^{\beta t}$, where $\beta=1 / \tau\left(\Lambda_{b}^{0}\right)-1 / \tau\left(\Xi_{b}^{-}\right)$. Many systematic uncertainties cancel to first order in the ratio, such as those associated with the time resolutions and relative acceptances.

The yields in each time bin are obtained using the results from the full fit with the signal shape parameters fixed. No dependence of the signal shapes on decay time is observed in simulated decays, as expected. The background shape parameters are also fixed, except for the combinatorial background shape parameter, and one of the $X_{b} \rightarrow X_{c} \rho$ shape parameters, which is seen to have a dependence on decay time. The signal yields in each of the time bins are shown in Table I. The relative acceptance, shown in Fig. 2, is obtained using simulated decays after applying all event selection criteria. The efficiency for reconstructing the $\Xi_{b}^{-} \rightarrow \Xi_{c}^{0} \pi^{-}$mode is about a factor of 2 lower than that of the $\Lambda_{b}^{0} \rightarrow \Lambda_{c}^{+} \pi^{-}$decay due to the extra particle in the final state and the lower average momentum of the finalstate particles. The relative efficiency $\epsilon\left(\Lambda_{b}^{0}\right) / \epsilon\left(\Xi_{b}^{-}\right)$is nearly uniform, with a gradual increase for decay times below 2 ps. This increase is expected, because the $\Lambda_{c}^{+}$ lifetime is about twice that of the $\Xi_{c}^{0}$ baryon, and the

TABLE I. Fitted yields of $\Lambda_{b}^{0} \rightarrow \Lambda_{c}^{+} \pi^{-}$and $\Xi_{b}^{-} \rightarrow \Xi_{c}^{0} \pi^{-}$in each time bin. Uncertainties are statistical only.

\begin{tabular}{lcc}
\hline \hline Decay time (ps) & $\Lambda_{b}^{0} \rightarrow \Lambda_{c}^{+} \pi^{-}$ & $\Xi_{b}^{-} \rightarrow \Xi_{c}^{0} \pi^{-}$ \\
\hline $0-1$ & $38989 \pm 212$ & $260 \pm 17$ \\
$1-2$ & $79402 \pm 299$ & $629 \pm 27$ \\
$2-3$ & $48979 \pm 233$ & $436 \pm 22$ \\
$3-4$ & $26010 \pm 169$ & $232 \pm 16$ \\
$4-6$ & $19651 \pm 147$ & $177 \pm 14$ \\
$6-9$ & $5794 \pm 79$ & $69 \pm 9$ \\
\hline \hline
\end{tabular}




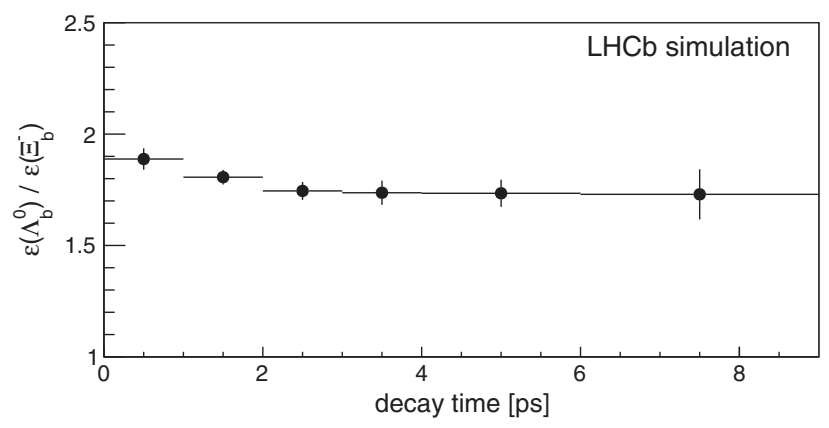

FIG. 2. Ratio of the $\Lambda_{b}^{0} \rightarrow \Lambda_{c}^{+} \pi^{-}$to the $\Xi_{b}^{-} \rightarrow \Xi_{c}^{0} \pi^{-}$selection efficiencies as a function of decay time. The uncertainties are due to the finite size of the simulated samples.

correspondingly larger impact parameters are favored by the software trigger and off-line selections, most notably when the $X_{b}$ decay time is small.

The ratios of corrected yields and the exponential fit are shown in Fig. 3. The points are displayed at the average time value in the bin assuming an exponential time distribution with mean $1.54 \mathrm{ps}$, which is the mean of the known $\Lambda_{b}^{0}$ and fitted $\Xi_{b}^{-}$lifetimes. Choosing either the $\Lambda_{b}^{0}$ or the fitted $\Xi_{b}^{-}$lifetime leads to a negligible change in the result. The fitted value is $\beta=0.0557 \pm 0.0160 \mathrm{ps}^{-1}$, where the uncertainty is statistical only. Using $\tau\left(\Lambda_{b}^{0}\right)=1.468 \pm$ $0.009 \pm 0.008 \mathrm{ps}$ [43], we find

$$
r_{\tau} \equiv \frac{\tau_{\Xi_{b}^{-}}}{\tau_{\Lambda_{b}^{0}}}=1.089 \pm 0.026(\text { stat })
$$

Several consistency checks are performed, including comparing the mass differences obtained from 7 versus $8 \mathrm{TeV}$ data, opposite magnet polarities, $X_{b}$ versus $\bar{X}_{b}$ samples, and different trigger selections. In all cases, the results are consistent with statistical fluctuations of independent samples. In addition, the analysis is carried out using $15500 B^{-} \rightarrow D^{0} \pi^{-}, \quad D^{0} \rightarrow K^{-} K^{+} \pi^{+} \pi^{-}$signal decays for normalization. The $\Xi_{b}^{-}$mass and lifetime results agree with the above values to better than 1 standard

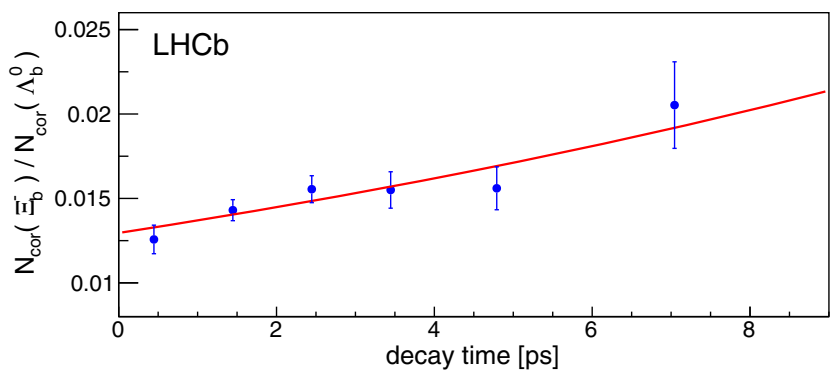

FIG. 3 (color online). Corrected yield ratio, $N_{\text {cor }}\left(\Xi_{b}^{-}\right) / N_{\text {cor }}\left(\Lambda_{b}^{0}\right)$ in bins of decay time, along with the exponential fit. The uncertainties are statistical only. deviation, considering only the uncertainty due to the $\Lambda_{b}^{0}$ and $B^{-}$masses and lifetimes.

The measurements of $M\left(\Xi_{b}^{-}\right)$and $\tau\left(\Xi_{b}^{-}\right)$are subject to systematic uncertainties, but the largest contributions cancel to first order in $\delta M$ and $r_{\tau}$. For the mass difference measurement, the effect of the momentum scale uncertainty of $0.03 \%$ [44] is investigated by shifting the momenta of all final-state particles in simulated decays by this amount, leading to an uncertainty on $\delta M$ of $0.08 \mathrm{MeV} / c^{2}$. Because the signal mode has one more particle than the normalization mode, the correction for energy loss in the detector material leads to an additional uncertainty of $0.06 \mathrm{MeV} / c^{2}$ [44]. Uncertainty due to the signal modeling is $0.06 \mathrm{MeV} / c^{2}$, obtained by shifting all fixed parameters by their uncertainties, and adding the shifts in $\delta M$ from the nominal value in quadrature. For the background model, several variations from the nominal fit are investigated, including (a) using a second-order polynomial to describe the combinatorial background, (b) allowing the fixed parameters in the partially reconstructed background to vary, (c) removing the $\Xi_{b}$ background component, (d) a $20 \%$ relative increase in the $\Xi_{b}^{-} \rightarrow \Xi_{c}^{0} K^{-}$cross feed, and (e) varying the fit range. The changes in $\delta M$ are added in quadrature to obtain the background uncertainty of $0.11 \mathrm{MeV} / c^{2}$. Adding all sources of uncertainty in quadrature leads to a systematic uncertainty in $\delta M$ of $0.16 \mathrm{MeV} / c^{2}$.

The largest source of systematic uncertainty in $r_{\tau}$ is the limited size of the simulated samples, which contributes an uncertainty of 0.010 . The simulated efficiencies are averaged over TOS and TIS events in the simulation, of which the former comprises $67 \%$ of the sample, compared to $57 \%$ in data. While the values of $r_{\tau}$ are statistically compatible between these two samples, if the efficiencies from simulation are reweighted to match the composition observed in data, a change in $r_{\tau}$ of 0.004 is found. This shift is assigned as a systematic uncertainty. Variation in the signal and background models lead to a negligible change in $r_{\tau}$. We also consider possible different performances of the BDT in data versus simulation by correcting the data with an efficiency obtained with a tighter BDT requirement. The difference of 0.001 is assigned as a systematic uncertainty. For the proton efficiency, we use the values obtained from simulation. By varying the proton PID requirements, a maximal change of 0.001 is found, which is assigned as a systematic uncertainty. To investigate possible effects due to the larger $\Lambda_{c}^{+}$lifetime (than the $\Xi_{c}^{0}$ ), we reject candidates with $c t$ larger than $150 \mu \mathrm{m}$. The difference of 0.003 from the nominal result is assigned as a systematic uncertainty. In total, the systematic uncertainty on $r_{\tau}$ is 0.011 .

In summary, we use a $p p$ collision data sample corresponding to $3.0 \mathrm{fb}^{-1}$ of integrated luminosity to improve the precision of the $\Xi_{b}^{-}$mass and lifetime by a factor of 2 over the previous best measurements. The resulting mass difference and relative lifetime are 


$$
\begin{aligned}
M\left(\Xi_{b}^{-}\right)-M\left(\Lambda_{b}^{0}\right) & =178.36 \pm 0.46 \pm 0.16 \mathrm{MeV} / c^{2}, \\
\frac{\tau_{\Xi_{b}^{-}}}{\tau_{\Lambda_{b}^{0}}} & =1.089 \pm 0.026 \pm 0.011,
\end{aligned}
$$

where the uncertainties are statistical and systematic, respectively. Using the measured $\Lambda_{b}^{0}$ mass [45] and lifetime [43], we find

$$
\begin{aligned}
M\left(\Xi_{b}^{-}\right) & =5797.72 \pm 0.46 \pm 0.16 \pm 0.26_{\Lambda_{b}^{0}} \mathrm{MeV} / c^{2}, \\
\tau_{\Xi_{b}^{-}} & =1.599 \pm 0.041 \pm 0.018 \pm 0.012_{\Lambda_{b}^{0}} \mathrm{ps},
\end{aligned}
$$

where the last uncertainty is due to the precision on the $\Lambda_{b}^{0}$ lifetime. Using the measurements of the $\Xi_{b}^{0}$ mass difference and relative lifetime, $M\left(\Xi_{b}^{0}\right)-M\left(\Lambda_{b}^{0}\right)=172.44 \pm 0.39 \pm$ $0.17 \mathrm{MeV} / c^{2}$ and $\tau_{\Xi_{b}^{0}} / \tau_{\Lambda_{b}^{0}}=1.006 \pm 0.018 \pm 0.010$ [15], we obtain

$$
\begin{aligned}
M\left(\Xi_{b}^{-}\right)-M\left(\Xi_{b}^{0}\right) & =5.92 \pm 0.60 \pm 0.23 \mathrm{MeV} / c^{2} \\
\frac{\tau_{\Xi_{b}^{-}}}{\tau_{\Xi_{b}^{0}}} & =1.083 \pm 0.032 \pm 0.016 .
\end{aligned}
$$

The measured isospin splitting between the $\Xi_{b}^{-}$and $\Xi_{b}^{0}$ baryons is consistent with the prediction in Ref. [22] of $6.24 \pm 0.21 \mathrm{MeV} / c^{2}$. The relative lifetime is 2.3 standard deviations larger than unity, giving a first indication that the $\Xi_{b}^{-}$baryon lifetime is larger than that of the $\Xi_{b}^{0}$ baryon. This result is consistent with the theoretical expectations of $\tau_{\Xi_{b}^{-}} / \tau_{\Xi_{b}^{0}}=1.05 \pm 0.07$ [12] and $\tau_{\Xi_{b}^{-}} / \tau_{\Lambda_{b}^{0}}=1.19_{-0.06}^{+0.07}[14]$, based on the HQE.

We express our gratitude to our colleagues in the CERN accelerator departments for the excellent performance of the LHC. We thank the technical and administrative staff at the LHCb institutes. We acknowledge support from CERN and from the national agencies: CAPES, CNPq, FAPERJ, and FINEP (Brazil); NSFC (China); CNRS/IN2P3 (France); BMBF, DFG, HGF, and MPG (Germany); SFI (Ireland); INFN (Italy); FOM and NWO (Netherlands); MNiSW and NCN (Poland); MEN/IFA (Romania); MinES and FANO (Russia); MinECo (Spain); SNSF and SER (Switzerland); NASU (Ukraine); STFC (United Kingdom); NSF (USA). The Tier1 computing centers are supported by IN2P3 (France), KIT and BMBF (Germany), INFN (Italy), NWO and SURF (Netherlands), PIC (Spain), GridPP (United Kingdom). We are indebted to the communities behind the multiple open source software packages on which we depend. We are also thankful for the computing resources and the access to software $R \& D$ tools provided by Yandex LLC (Russia). Individual groups or members have received support from EPLANET, Marie Skłodowska-Curie Actions, and ERC (European Union), Conseil général de Haute-Savoie, Labex ENIGMASS, and OCEVU, Région Auvergne (France), RFBR (Russia), XuntaGal and GENCAT (Spain), Royal Society and Royal Commission for the Exhibition of 1851 (United Kingdom).

[1] V. A. Khoze and M. A. Shifman, Sov. Phys. Usp. 26, 387 (1983).

[2] I. I. Bigi and N. G. Uraltsev, Phys. Lett. B 280, 271 (1992).

[3] I. I. Bigi, N. G. Uraltsev, and A. I. Vainshtein, Phys. Lett. B 293, 430 (1992).

[4] B. Blok and M. Shifman, Nucl. Phys. B399, 441 (1993).

[5] B. Blok and M. Shifman, Nucl. Phys. B399, 459 (1993).

[6] M. Neubert, Adv. Ser. Dir. High Energy Phys. 15, 239 (1998).

[7] N. Uraltsev, in Heavy Flavour Physics: A Probe of Nature's Grand Design, Proceedings of the International School of Physics "Enrico Fermi," Course CXXXVII, Varenna, 1997 (IOS Press, Amsterdam, 1998), p. 329.

[8] I. I. Bigi, arXiv:hep-ph/9508408.

[9] K. A. Olive et al. (Particle Data Group), Chin. Phys. C38, 090001 (2014).

[10] R. Aaij et al. (LHCb Collaboration), Phys. Rev. Lett. 113, 172001 (2014).

[11] S. Stone, arXiv:1406.6497.

[12] A. Lenz, arXiv:1405.3601, invited contribution to the Kolya Uraltsev Memorial Book.

[13] K. G. Wilson, Phys. Rev. D 10, 2445 (1974).

[14] M. Voloshin, Phys. Rev. D 61, 074026 (2000).

[15] R. Aaij et al. (LHCb Collaboration), Phys. Rev. Lett. 113, 032001 (2014).

[16] R. Aaij et al. (LHCb Collaboration), Phys. Lett. B 736, 154 (2014).

[17] T. Aaltonen et al. (CDF Collaboration), Phys. Rev. D 89, 072014 (2014).

[18] D. Ebert, R. N. Faustov, and V. O. Galkin, Phys. Rev. D 72, 034026 (2005).

[19] E. E. Jenkins, Phys. Rev. D 77, 034012 (2008).

[20] X. Liu, Hua-Xing Chen, Yan-Rui Liu, Atsushi Hosaka, and Shi-Lin Zhu, Phys. Rev. D 77, 014031 (2008).

[21] R. Roncaglia, D. B. Lichtenberg, and E. Predazzi, Phys. Rev. D 52, 1722 (1995).

[22] M. Karliner, B. Keren-Zur, H. J. Lipkin, and J. L. Rosner, Ann. Phys. (N.Y.) 324, 2 (2009).

[23] M. Karliner, Nucl. Phys. B, Proc. Suppl. 187, 21 (2009).

[24] W. Roberts and M. Pervin, Int. J. Mod. Phys. A 23, 2817 (2008).

[25] Z. Ghalenovi and A. Akbar Rajabi, Eur. Phys. J. Plus 127, 141 (2012).

[26] B. Patel, A. K. Rai, and P. C. Vinodkumar, J. Phys. G 35, 065001 (2008).

[27] B. Patel, A. K. Rai, and P. C. Vinodkumar, Pramana 70, 797 (2008).

[28] R. Aaij et al. (LHCb Collaboration), Phys. Rev. Lett. 110, 182001 (2013).

[29] A. A. Alves, Jr. et al. (LHCb Collaboration), JINST 3, S08005 (2008).

[30] M. Adinolfi et al., Eur. Phys. J. C 73, 2431 (2013).

[31] A. A. Alves, Jr. et al., JINST 8, P02022 (2013). 
[32] R. Aaij et al., JINST 8, P04022 (2013).

[33] V. V. Gligorov and M. Williams, JINST 8, P02013 (2013).

[34] T. Sjöstrand, S. Mrenna, and P. Skands, J. High Energy Phys. 05 (2006) 026; T. Sjöstrand, S. Mrenna, and P. Skands, Comput. Phys. Commun. 178, 852 (2008).

[35] I. Belyaev et al., Nuclear Science Symposium Conference Record (NSS/MIC) IEEE, 1155 (2010).

[36] D. J. Lange, Nucl. Instrum. Methods Phys. Res., Sect. A 462, 152 (2001).

[37] P. Golonka and Z. Was, Eur. Phys. J. C 45, 97 (2006).

[38] J. Allison et al. (GEANT4 Collaboration), IEEE Trans. Nucl. Sci. 53, 270 (2006); S. Agostinelli et al. (GEANT4 Collaboration), Nucl. Instrum. Methods Phys. Res., Sect. A 506, 250 (2003).
[39] M. Clemencic, G. Corti, S. Easo, C. R Jones, S. Miglioranzi, M. Pappagallo, and P Robbe, J. Phys. Conf. Ser. 331, 032023 (2011).

[40] L. Breiman, J. H. Friedman, R. A. Olshen, and C. J. Stone, Classification and regression trees (Wadsworth Intl. Group, Belmont, CA, 1984).

[41] Y. Freund and R. E. Schapire, J. Comput. Syst. Sci. 55, 119 (1997).

[42] T. Skwarnicki, Ph.D. thesis, Institute of Nuclear Physics, Krakow, 1986, DESY-F31-86-02.

[43] R. Aaij et al. (LHCb Collaboration), Phys. Lett. B 734, 122 (2014).

[44] R. Aaij et al. (LHCb Collaboration), J. High Energy Phys. 06 (2013) 065.

[45] R. Aaij et al. (LHCb Collaboration), Phys. Rev. Lett. 112, 202001 (2014).

R. Aaij, ${ }^{41}$ B. Adeva, ${ }^{37}$ M. Adinolfi,${ }^{46}$ A. Affolder, ${ }^{52}$ Z. Ajaltouni, ${ }^{5}$ S. Akar, ${ }^{6}$ J. Albrecht, ${ }^{9}$ F. Alessio, ${ }^{38}$ M. Alexander, ${ }^{51}$ S. Ali, ${ }^{41}$ G. Alkhazov, ${ }^{30}$ P. Alvarez Cartelle, ${ }^{37}$ A. A. Alves Jr., ${ }^{25,38}$ S. Amato, ${ }^{2}$ S. Amerio, ${ }^{22}$ Y. Amhis, ${ }^{7}$ L. An, ${ }^{3}$

L. Anderlini, ${ }^{17, a}$ J. Anderson, ${ }^{40}$ R. Andreassen, ${ }^{57}$ M. Andreotti, ${ }^{16, b}$ J. E. Andrews,${ }^{58}$ R. B. Appleby, ${ }^{54}$

O. Aquines Gutierrez, ${ }^{10}$ F. Archilli, ${ }^{38}$ A. Artamonov, ${ }^{35}$ M. Artuso, ${ }^{59}$ E. Aslanides, ${ }^{6}$ G. Auriemma, ${ }^{25, c}$ M. Baalouch, ${ }^{5}$ S. Bachmann, ${ }^{11}$ J. J. Back, ${ }^{48}$ A. Badalov,${ }^{36}$ C. Baesso, ${ }^{60}$ W. Baldini, ${ }^{16}$ R. J. Barlow ${ }^{54}$ C. Barschel,${ }^{38}$ S. Barsuk, W. Barter, ${ }^{47}$ V. Batozskaya, ${ }^{28}$ V. Battista, ${ }^{39}$ A. Bay, ${ }^{39}$ L. Beaucourt, ${ }^{4}$ J. Beddow, ${ }^{51}$ F. Bedeschi, ${ }^{23}$ I. Bediaga, ${ }^{1}$ S. Belogurov, ${ }^{31}$ K. Belous,${ }^{35}$ I. Belyaev, ${ }^{31}$ E. Ben-Haim, ${ }^{8}$ G. Bencivenni, ${ }^{18}$ S. Benson, ${ }^{38}$ J. Benton, ${ }^{46}$ A. Berezhnoy, ${ }^{32}$ R. Bernet, ${ }^{40}$ M.-O. Bettler, ${ }^{47}$ M. van Beuzekom, ${ }^{41}$ A. Bien, ${ }^{11}$ S. Bifani, ${ }^{45}$ T. Bird ${ }^{54}$ A. Bizzeti, ${ }^{17, d}$ P. M. Bjørnstad,${ }^{54}$ T. Blake, ${ }^{48}$ F. Blanc, ${ }^{39}$ J. Blouw, ${ }^{10}$ S. Blusk, ${ }^{59}$ V. Bocci, ${ }^{25}$ A. Bondar, ${ }^{34}$ N. Bondar,${ }^{30,38}$ W. Bonivento, ${ }^{15,38}$ S. Borghi, ${ }^{54}$ A. Borgia, ${ }^{59}$ M. Borsato, ${ }^{7}$ T. J. V. Bowcock,${ }^{52}$ E. Bowen, ${ }^{40}$ C. Bozzi, ${ }^{16}$ T. Brambach,,${ }^{9}$ D. Brett, ${ }^{54}$ M. Britsch, ${ }^{10}$ T. Britton, ${ }^{59}$ J. Brodzicka, ${ }^{54}$ N. H. Brook, ${ }^{46}$ H. Brown, ${ }^{52}$ A. Bursche, ${ }^{40}$ J. Buytaert, ${ }^{38}$ S. Cadeddu,${ }^{15}$ R. Calabrese, ${ }^{16, b}$ M. Calvi, ${ }^{20, e}$ M. Calvo Gomez, ${ }^{36, \mathrm{f}}$ P. Campana, ${ }^{18}$ D. Campora Perez,${ }^{38}$ A. Carbone, ${ }^{14, \mathrm{~g}}$ G. Carboni, ${ }^{24, \mathrm{~h}}$ R. Cardinale, ${ }^{19,38, \mathrm{i}}$ A. Cardini, ${ }^{15}$ L. Carson, ${ }^{50}$ K. Carvalho Akiba, ${ }^{2}$ G. Casse, ${ }^{52}$ L. Cassina, ${ }^{20}$ L. Castillo Garcia,${ }^{38}$ M. Cattaneo, ${ }^{38}$ Ch. Cauet, ${ }^{9}$ R. Cenci, ${ }^{23}$ M. Charles, ${ }^{8}$ Ph. Charpentier, ${ }^{38}$ M. Chefdeville, ${ }^{4}$ S. Chen, ${ }^{54}$ S.-F. Cheung, ${ }^{55}$ N. Chiapolini ${ }^{40}$ M. Chrzaszcz, ${ }^{40,26}$ X. Cid Vidal, ${ }^{38}$ G. Ciezarek, ${ }^{53}$ P. E. L. Clarke, ${ }^{50}$ M. Clemencic, ${ }^{38}$ H. V. Cliff, ${ }^{47}$ J. Closier, ${ }^{38}$ V. Coco, ${ }^{38}$ J. Cogan, ${ }^{6}$ E. Cogneras, ${ }^{5}$ V. Cogoni,${ }^{15}$ L. Cojocariu, ${ }^{29}$ G. Collazuol, ${ }^{22}$ P. Collins,${ }^{38}$ A. Comerma-Montells, ${ }^{11}$ A. Contu,,${ }^{15,38}$ A. Cook, ${ }^{46}$ M. Coombes, ${ }^{46}$ S. Coquereau, ${ }^{8}$ G. Corti,${ }^{38}$ M. Corvo, ${ }^{16, b}$ I. Counts, ${ }^{56}$ B. Couturier, ${ }^{38}$ G. A. Cowan, ${ }^{50}$ D. C. Craik, ${ }^{48}$ M. Cruz Torres, ${ }^{60}$ S. Cunliffe, ${ }^{53}$ R. Currie,${ }^{53}$ C. D'Ambrosio, ${ }^{38}$ J. Dalseno,${ }^{46}$ P. David,${ }^{8}$ P. N. Y. David, ${ }^{41}$ A. Davis ${ }^{57}$ K. De Bruyn, ${ }^{41}$ S. De Capua,${ }^{54}$ M. De Cian,,${ }^{11}$ J. M. De Miranda, ${ }^{1}$ L. De Paula, ${ }^{2}$ W. De Silva,${ }^{57}$ P. De Simone, ${ }^{18}$ C.-T. Dean,${ }^{51}$ D. Decamp, ${ }^{4}$ M. Deckenhoff, ${ }^{9}$ L. Del Buono,${ }^{8}$ N. Déléage, ${ }^{4}$ D. Derkach, ${ }^{55}$ O. Deschamps, ${ }^{5}$ F. Dettori,${ }^{38}$ A. Di Canto, ${ }^{38}$ H. Dijkstra, ${ }^{38}$ S. Donleavy, ${ }^{52}$ F. Dordei,${ }^{11}$ M. Dorigo, ${ }^{39}$ A. Dosil Suárez,${ }^{37}$ D. Dossett, ${ }^{48}$ A. Dovbnya ${ }^{43}$ K. Dreimanis, ${ }^{52}$ G. Dujany, ${ }^{54}$ F. Dupertuis, ${ }^{39}$ P. Durante,${ }^{38}$ R. Dzhelyadin, ${ }^{35}$ A. Dziurda, ${ }^{26}$ A. Dzyuba, ${ }^{30}$ S. Easo, ${ }^{49,38}$ U. Egede, ${ }^{53}$ V. Egorychev, ${ }^{31}$ S. Eidelman, ${ }^{34}$ S. Eisenhardt, ${ }^{50}$ U. Eitschberger, ${ }^{9}$ R. Ekelhof, ${ }^{9}$ L. Eklund,${ }^{51}$ I. El Rifai, ${ }^{5}$ Ch. Elsasser, ${ }^{40}$ S. Ely, ${ }^{59}$ S. Esen, ${ }^{11}$ H.-M. Evans,${ }^{47}$ T. Evans, ${ }^{55}$ A. Falabella, ${ }^{14}$ C. Färber, ${ }^{11}$ C. Farinelli, ${ }^{41}$ N. Farley, ${ }^{45}$ S. Farry, ${ }^{52}$ RF Fay, ${ }^{52}$ D. Ferguson ${ }^{50}$ V. Fernandez Albor, ${ }^{37}$ F. Ferreira Rodrigues, ${ }^{1}$ M. Ferro-Luzzi, ${ }^{38}$ S. Filippov, ${ }^{33}$ M. Fiore, ${ }^{16, b}$ M. Fiorini, ${ }^{16, b}$ M. Firlej, ${ }^{27}$ C. Fitzpatrick, ${ }^{39}$ T. Fiutowski, ${ }^{27}$ P. Fol,${ }^{53}$ M. Fontana,${ }^{10}$ F. Fontanelli,,${ }^{19, i}$ R. Forty, ${ }^{38}$ O. Francisco, ${ }^{2}$ M. Frank,${ }^{38}$ C. Frei, ${ }^{38}$ M. Frosini, ${ }^{17, a}$ J. Fu, ${ }^{21,38}$ E. Furfaro, ${ }^{24, h}$ A. Gallas Torreira, ${ }^{37}$ D. Galli, ${ }^{14, g}$ S. Gallorini, ${ }^{22,38}$ S. Gambetta, ${ }^{19, i}$ M. Gandelman, ${ }^{2}$ P. Gandini, ${ }^{59}$ Y. Gao, ${ }^{3}$ J. García Pardiñas, ${ }^{37}$ J. Garofoli, ${ }^{59}$ J. Garra Tico, ${ }^{47}$ L. Garrido, ${ }^{36}$ D. Gascon, ${ }^{36}$ C. Gaspar, ${ }^{38}$ R. Gauld ${ }^{55}$ L. Gavardi, ${ }^{9}$ A. Geraci, ${ }^{21, j}$ E. Gersabeck, ${ }^{11}$ M. Gersabeck,${ }^{54}$ T. Gershon, ${ }^{48}$ Ph. Ghez, ${ }^{4}$ A. Gianelle, ${ }^{22}$ S. Gianì ${ }^{39}$ V. Gibson, ${ }^{47}$ L. Giubega, ${ }^{29}$ V. V. Gligorov, ${ }^{38}$ C. Göbel, ${ }^{60}$ D. Golubkov, ${ }^{31}$ A. Golutvin, ${ }^{53,31,38}$ A. Gomes, ${ }^{1, k}$ C. Gotti, ${ }^{20}$ M. Grabalosa Gándara, ${ }^{5}$ R. Graciani Diaz, ${ }^{36}$ L. A. Granado Cardoso, ${ }^{38}$ E. Graugés,${ }^{36}$ E. Graverini, ${ }^{40}$ G. Graziani, ${ }^{17}$ A. Grecu, ${ }^{29}$ E. Greening, ${ }^{55}$ S. Gregson, ${ }^{47}$ P. Griffith, ${ }^{45}$ L. Grillo, ${ }^{11}$ O. Grünberg, ${ }^{63}$ B. Gui,${ }^{59}$ E. Gushchin, ${ }^{33}$ Yu. Guz, ${ }^{35,38}$ T. Gys, ${ }^{38}$ C. Hadjivasiliou, ${ }^{59}$ G. Haefeli, ${ }^{39}$ C. Haen,${ }^{38}$ S. C. Haines,${ }^{47}$ S. Hall,${ }^{53}$ B. Hamilton,${ }^{58}$ T. Hampson, ${ }^{46}$ 
X. Han, ${ }^{11}$ S. Hansmann-Menzemer, ${ }^{11}$ N. Harnew, ${ }^{55}$ S. T. Harnew, ${ }^{46}$ J. Harrison,${ }^{54}$ J. He, ${ }^{38}$ T. Head ${ }^{38}$ V. Heijne, ${ }^{41}$ K. Hennessy, ${ }^{52}$ P. Henrard, ${ }^{5}$ L. Henry, ${ }^{8}$ J. A. Hernando Morata ${ }^{37}$ E. van Herwijnen, ${ }^{38}$ M. He $\beta,{ }^{63}$ A. Hicheur, ${ }^{2}$ D. Hill, ${ }^{55}$ M. Hoballah, ${ }^{5}$ C. Hombach, ${ }^{54}$ W. Hulsbergen, ${ }^{41}$ P. Hunt,${ }^{55}$ N. Hussain, ${ }^{55}$ D. Hutchcroft, ${ }^{52}$ D. Hynds,${ }^{51}$ M. Idzik, ${ }^{27}$ P. Ilten, ${ }^{56}$ R. Jacobsson, ${ }^{38}$ A. Jaeger, ${ }^{11}$ J. Jalocha, ${ }^{55}$ E. Jans, ${ }^{41}$ P. Jaton, ${ }^{39}$ A. Jawahery, ${ }^{58}$ F. Jing, ${ }^{3}$ M. John, ${ }^{55}$ D. Johnson, ${ }^{38}$ C. R. Jones, ${ }^{47}$ C. Joram, ${ }^{38}$ B. Jost,${ }^{38}$ N. Jurik ${ }^{59}$ S. Kandybei, ${ }^{43}$ W. Kanso, ${ }^{6}$ M. Karacson,${ }^{38}$ T. M. Karbach, ${ }^{38}$ S. Karodia,${ }^{51}$ M. Kelsey, ${ }^{59}$ I. R. Kenyon, ${ }^{45}$ T. Ketel, ${ }^{42}$ B. Khanji, ${ }^{20,38}$ C. Khurewathanakul, ${ }^{39}$ S. Klaver, ${ }^{54}$ K. Klimaszewski, ${ }^{28}$ O. Kochebina, ${ }^{7}$ M. Kolpin, ${ }^{11}$ I. Komarov, ${ }^{39}$ R. F. Koopman, ${ }^{42}$ P. Koppenburg, ${ }^{41,38}$ M. Korolev, ${ }^{32}$ A. Kozlinskiy, ${ }^{41}$ L. Kravchuk, ${ }^{33}$ K. Kreplin, ${ }^{11}$ M. Kreps, ${ }^{48}$ G. Krocker, ${ }^{11}$ P. Krokovny, ${ }^{34}$ F. Kruse, ${ }^{9}$ W. Kucewicz, ${ }^{26,1}$ M. Kucharczyk,${ }^{20,26, e}$ V. Kudryavtsev, ${ }^{34}$ K. Kurek, ${ }^{28}$ T. Kvaratskheliya, ${ }^{31}$ V. N. La Thi, ${ }^{39}$ D. Lacarrere, ${ }^{38}$ G. Lafferty, ${ }^{54}$ A. Lai, ${ }^{15}$ D. Lambert,${ }^{50}$ R. W. Lambert, ${ }^{42}$ G. Lanfranchi, ${ }^{18}$ C. Langenbruch, ${ }^{48}$ B. Langhans, ${ }^{38}$ T. Latham, ${ }^{48}$ C. Lazzeroni, ${ }^{45}$ R. Le Gac, ${ }^{6}$ J. van Leerdam, ${ }^{41}$ J.-P. Lees, ${ }^{4}$ R. Lefèvre, ${ }^{5}$ A. Leflat,${ }^{32}$ J. Lefrançois, ${ }^{7}$ S. Leo, ${ }^{23}$ O. Leroy, ${ }^{6}$ T. Lesiak, ${ }^{26}$ B. Leverington, ${ }^{11}$ Y. Li, ${ }^{3}$ T. Likhomanenko, ${ }^{64}$ M. Liles, ${ }^{52}$ R. Lindner, ${ }^{38}$ C. Linn, ${ }^{38}$ F. Lionetto, ${ }^{40}$ B. Liu, ${ }^{15}$ S. Lohn, ${ }^{38}$ I. Longstaff, ${ }^{51}$ J. H. Lopes, ${ }^{2}$ N. Lopez-March, ${ }^{39}$ P. Lowdon, ${ }^{40}$ D. Lucchesi, ${ }^{22, m}$ H. Luo ${ }^{50}$ A. Lupato, ${ }^{22}$ E. Luppi, ${ }^{16, b}$ O. Lupton, ${ }^{55}$ F. Machefert, ${ }^{7}$ I. V. Machikhiliyan, ${ }^{31}$ F. Maciuc,${ }^{29}$ O. Maev,${ }^{30}$ S. Malde, ${ }^{55}$ A. Malinin, ${ }^{64}$ G. Manca, ${ }^{15, \mathrm{n}}$ G. Mancinelli, ${ }^{6}$ A. Mapelli, ${ }^{38}$ J. Maratas, ${ }^{5}$ J. F. Marchand ${ }^{4}$ U. Marconi,${ }^{14}$ C. Marin Benito, ${ }^{36}$ P. Marino, ${ }^{23,0}$ R. Märki, ${ }^{39}$ J. Marks, ${ }^{11}$ G. Martellotti, ${ }^{25}$ A. Martín Sánchez, ${ }^{7}$ M. Martinelli, ${ }^{39}$ D. Martinez Santos, ${ }^{42,38}$ F. Martinez Vidal, ${ }^{65}$ D. Martins Tostes, ${ }^{2}$ A. Massafferri, ${ }^{1}$ R. Matev,${ }^{38}$ Z. Mathe,${ }^{38}$ C. Matteuzzi, ${ }^{20}$ B. Maurin, ${ }^{39}$ A. Mazurov, ${ }^{45}$ M. McCann, ${ }^{53}$ J. McCarthy, ${ }^{45}$ A. McNab, ${ }^{54}$ R. McNulty, ${ }^{12}$ B. McSkelly,${ }^{52}$ B. Meadows, ${ }^{57}$ F. Meier, ${ }^{9}$ M. Meissner, ${ }^{11}$ M. Merk, ${ }^{41}$ D. A. Milanes ${ }^{62}$

M.-N. Minard, ${ }^{4}$ N. Moggi, ${ }^{14}$ J. Molina Rodriguez, ${ }^{60}$ S. Monteil,${ }^{5}$ M. Morandin, ${ }^{22}$ P. Morawski, ${ }^{27}$ A. Mordà, ${ }^{6}$

M. J. Morello, ${ }^{23,0}$ J. Moron, ${ }^{27}$ A.-B. Morris,${ }^{50}$ R. Mountain, ${ }^{59}$ F. Muheim, ${ }^{50}$ K. Müller, ${ }^{40}$ M. Mussini, ${ }^{14}$ B. Muster, ${ }^{39}$ P. Naik, ${ }^{46}$ T. Nakada, ${ }^{39}$ R. Nandakumar, ${ }^{49}$ I. Nasteva, ${ }^{2}$ M. Needham, ${ }^{50}$ N. Neri, ${ }^{21}$ S. Neubert, ${ }^{38}$ N. Neufeld,${ }^{38}$ M. Neuner, ${ }^{11}$

A. D. Nguyen, ${ }^{39}$ T. D. Nguyen, ${ }^{39}$ C. Nguyen-Mau, ${ }^{39, p}$ M. Nicol, ${ }^{7}$ V. Niess, ${ }^{5}$ R. Niet, ${ }^{9}$ N. Nikitin, ${ }^{32}$ T. Nikodem, ${ }^{11}$ A. Novoselov, ${ }^{35}$ D. P. O'Hanlon, ${ }^{48}$ A. Oblakowska-Mucha, ${ }^{27,38}$ V. Obraztsov, ${ }^{35}$ S. Oggero, ${ }^{41}$ S. Ogilvy, ${ }^{51}$ O. Okhrimenko, ${ }^{44}$ R. Oldeman, ${ }^{15, n}$ C. J. G. Onderwater, ${ }^{66}$ M. Orlandea, ${ }^{29}$ J. M. Otalora Goicochea, ${ }^{2}$ A. Otto, ${ }^{38}$ P. Owen, ${ }^{53}$ A. Oyanguren, ${ }^{65}$ B. K. Pal, ${ }^{59}$ A. Palano, ${ }^{13, q}$ F. Palombo,${ }^{21, \mathrm{r}}$ M. Palutan, ${ }^{18}$ J. Panman, ${ }^{38}$ A. Papanestis, ${ }^{49,38}$ M. Pappagallo, ${ }^{51}$ L. L. Pappalardo, ${ }^{16, \mathrm{~b}}$ C. Parkes,${ }^{54}$ C. J. Parkinson,,${ }^{9,45}$ G. Passaleva,${ }^{17}$ G. D. Patel,${ }^{52}$ M. Patel,${ }^{53}$ C. Patrignani, ${ }^{19, \mathrm{i}}$ A. Pearce,${ }^{54}$ A. Pellegrino, ${ }^{41}$ M. Pepe Altarelli, ${ }^{38}$ S. Perazzini, ${ }^{14, g}$ P. Perret,${ }^{5}$ M. Perrin-Terrin, ${ }^{6}$ L. Pescatore, ${ }^{45}$ E. Pesen, ${ }^{67}$ K. Petridis, ${ }^{53}$ A. Petrolini ${ }^{19, i}$ E. Picatoste Olloqui ${ }^{36}$ B. Pietrzyk,${ }^{4}$ T. Pilař, ${ }^{48}$ D. Pinci, ${ }^{25}$ A. Pistone, ${ }^{19}$ S. Playfer, ${ }^{50}$ M. Plo Casasus, ${ }^{37}$ F. Polci, ${ }^{8}$ A. Poluektov, ${ }^{48,34}$ E. Polycarpo, ${ }^{2}$ A. Popov, ${ }^{35}$ D. Popov, ${ }^{10}$ B. Popovici ${ }^{29}$ C. Potterat, ${ }^{2}$ E. Price, ${ }^{46}$ J. D. Price, ${ }^{52}$ J. Prisciandaro, ${ }^{39}$ A. Pritchard ${ }^{52}$ C. Prouve, ${ }^{46}$ V. Pugatch, ${ }^{44}$ A. Puig Navarro, ${ }^{39}$ G. Punzi, ${ }^{23, s}$ W. Qian, ${ }^{4}$ B. Rachwal, ${ }^{26}$ J. H. Rademacker, ${ }^{46}$ B. Rakotomiaramanana, ${ }^{39}$ M. Rama, ${ }^{18}$ M. S. Rangel,${ }^{2}$ I. Raniuk,${ }^{43}$ N. Rauschmayr, ${ }^{38}$ G. Raven, ${ }^{42}$

F. Redi, ${ }^{53}$ S. Reichert, ${ }^{54}$ M. M. Reid, ${ }^{48}$ A. C. dos Reis, ${ }^{1}$ S. Ricciardi, ${ }^{49}$ S. Richards, ${ }^{46}$ M. Rihl,${ }^{38}$ K. Rinnert, ${ }^{52}$

V. Rives Molina ${ }^{36}$ P. Robbe, ${ }^{7}$ A. B. Rodrigues, ${ }^{1}$ E. Rodrigues, ${ }^{54}$ P. Rodriguez Perez,${ }^{54}$ S. Roiser, ${ }^{38}$ V. Romanovsky, ${ }^{35}$ A. Romero Vidal, ${ }^{37}$ M. Rotondo, ${ }^{22}$ J. Rouvinet, ${ }^{39}$ T. Ruf, ${ }^{38}$ H. Ruiz, ${ }^{36}$ P. Ruiz Valls, ${ }^{65}$ J. J. Saborido Silva, ${ }^{37}$ N. Sagidova, ${ }^{30}$ P. Sail,${ }^{51}$ B. Saitta, ${ }^{15, \mathrm{n}}$ V. Salustino Guimaraes, ${ }^{2}$ C. Sanchez Mayordomo ${ }^{65}$ B. Sanmartin Sedes, ${ }^{37}$ R. Santacesaria, ${ }^{25}$

C. Santamarina Rios, ${ }^{37}$ E. Santovetti, ${ }^{24, \mathrm{~h}}$ A. Sarti, ${ }^{18, t}$ C. Satriano, ${ }^{25, \mathrm{c}}$ A. Satta, ${ }^{24}$ D. M. Saunders, ${ }^{46}$ D. Savrina, ${ }^{31,32}$ M. Schiller, ${ }^{42}$ H. Schindler, ${ }^{38}$ M. Schlupp, ${ }^{9}$ M. Schmelling, ${ }^{10}$ B. Schmidt, ${ }^{38}$ O. Schneider, ${ }^{39}$ A. Schopper, ${ }^{38}$ M. Schubiger, ${ }^{39}$ M.-H. Schune, ${ }^{7}$ R. Schwemmer, ${ }^{38}$ B. Sciascia, ${ }^{18}$ A. Sciubba, ${ }^{25}$ A. Semennikov, ${ }^{31}$ I. Sepp, ${ }^{53}$ N. Serra, ${ }^{40}$ J. Serrano, ${ }^{6}$ L. Sestini, ${ }^{22}$ P. Seyfert, ${ }^{11}$ M. Shapkin, ${ }^{35}$ I. Shapoval, ${ }^{16,43, b}$ Y. Shcheglov, ${ }^{30}$ T. Shears, ${ }^{52}$ L. Shekhtman, ${ }^{34}$ V. Shevchenko, ${ }^{64}$ A. Shires, ${ }^{9}$ R. Silva Coutinho, ${ }^{48}$ G. Simi,${ }^{22}$ M. Sirendi, ${ }^{47}$ N. Skidmore, ${ }^{46}$ I. Skillicorn, ${ }^{51}$ T. Skwarnicki,${ }^{59}$ N. A. Smith, ${ }^{52}$ E. Smith, ${ }^{55,49}$ E. Smith,${ }^{53}$ J. Smith, ${ }^{47}$ M. Smith,${ }^{54}$ H. Snoek, ${ }^{41}$ M. D. Sokoloff, ${ }^{57}$ F. J. P. Soler, ${ }^{51}$ F. Soomro, ${ }^{39}$ D. Souza, ${ }^{46}$ B. Souza De Paula, ${ }^{2}$ B. Spaan, ${ }^{9}$ P. Spradlin, ${ }^{51}$ S. Sridharan, ${ }^{38}$ F. Stagni, ${ }^{38}$ M. Stahl, ${ }^{11}$ S. Stahl, ${ }^{11}$ O. Steinkamp, ${ }^{40}$ O. Stenyakin, ${ }^{35}$ S. Stevenson, ${ }^{55}$ S. Stoica, ${ }^{29}$ S. Stone, ${ }^{59}$ B. Storaci,${ }^{40}$ S. Stracka, ${ }^{23}$ M. Straticiuc,${ }^{29}$ U. Straumann, ${ }^{40}$ R. Stroili, ${ }^{22}$ V. K. Subbiah, ${ }^{38}$ L. Sun, ${ }^{57}$ W. Sutcliffe, ${ }^{53}$ K. Swientek, ${ }^{27}$ S. Swientek, ${ }^{9}$ V. Syropoulos, ${ }^{42}$ M. Szczekowski, ${ }^{28}$ P. Szczypka, ${ }^{39,38}$ T. Szumlak, ${ }^{27}$ S. T'Jampens, ${ }^{4}$ M. Teklishyn, ${ }^{7}$ G. Tellarini, ${ }^{16, b}$ F. Teubert, ${ }^{38}$ C. Thomas,${ }^{55}$ E. Thomas,${ }^{38}$ J. van Tilburg, ${ }^{41}$ V. Tisserand, ${ }^{4}$ M. Tobin, ${ }^{39}$ J. Todd, ${ }^{57}$ S. Tolk, ${ }^{42}$ L. Tomassetti, ${ }^{16, b}$ D. Tonelli, ${ }^{38}$ S. Topp-Joergensen,${ }^{55}$ N. Torr, ${ }^{55}$ E. Tournefier, ${ }^{4}$ S. Tourneur, ${ }^{39}$ M. T. Tran,${ }^{39}$ M. Tresch, ${ }^{40}$ A. Trisovic, ${ }^{38}$ A. Tsaregorodtsev, ${ }^{6}$ P. Tsopelas, ${ }^{41}$ N. Tuning, ${ }^{41}$ M. Ubeda Garcia, ${ }^{38}$ A. Ukleja, ${ }^{28}$ A. Ustyuzhanin, ${ }^{64}$ U. Uwer, ${ }^{11}$ C. Vacca, ${ }^{15}$ V. Vagnoni, ${ }^{14}$ G. Valenti, ${ }^{14}$ A. Vallier, ${ }^{7}$ R. Vazquez Gomez, ${ }^{18}$ P. Vazquez Regueiro, ${ }^{37}$ C. Vázquez Sierra, ${ }^{37}$ S. Vecchi, ${ }^{16}$ J. J. Velthuis, ${ }^{46}$ M. Veltri, ${ }^{17, u}$ 
G. Veneziano, ${ }^{39}$ M. Vesterinen, ${ }^{11}$ B. Viaud,${ }^{7}$ D. Vieira, ${ }^{2}$ M. Vieites Diaz ${ }^{37}$ X. Vilasis-Cardona, ${ }^{36, f}$ A. Vollhardt,${ }^{40}$ D. Volyanskyy, ${ }^{10}$ D. Voong, ${ }^{46}$ A. Vorobyev,${ }^{30}$ V. Vorobyev,${ }^{34}$ C. Voß, ${ }^{63}$ J. A. de Vries, ${ }^{41}$ R. Waldi, ${ }^{63}$ C. Wallace, ${ }^{48}$ R. Wallace, ${ }^{12}$ J. Walsh, ${ }^{23}$ S. Wandernoth, ${ }^{11}$ J. Wang, ${ }^{59}$ D. R. Ward ${ }^{47}$ N. K. Watson, ${ }^{45}$ D. Websdale ${ }^{53}$ M. Whitehead ${ }^{48}$ J. Wicht ${ }^{38}$ D. Wiedner, ${ }^{11}$ G. Wilkinson, ${ }^{55,38}$ M. P. Williams, ${ }^{45}$ M. Williams,${ }^{56}$ H. W. Wilschut, ${ }^{66}$ F. F. Wilson, ${ }^{49}$ J. Wimberley, ${ }^{58}$ J. Wishahi, ${ }^{9}$ W. Wislicki, ${ }^{28}$ M. Witek, ${ }^{26}$ G. Wormser, ${ }^{7}$ S. A. Wotton, ${ }^{47}$ S. Wright, ${ }^{47}$ K. Wyllie, ${ }^{38}$ Y. Xie,${ }^{61}$ Z. Xing, ${ }^{59}$ Z. Xu, ${ }^{39}$ Z. Yang, ${ }^{3}$ X. Yuan, ${ }^{3}$ O. Yushchenko, ${ }^{35}$ M. Zangoli, ${ }^{14}$ M. Zavertyaev, ${ }^{10, v}$ L. Zhang, ${ }^{59}$ W. C. Zhang, ${ }^{12}$ Y. Zhang, ${ }^{3}$ A. Zhelezov, ${ }^{11}$ A. Zhokhov ${ }^{31}$ and L. Zhong ${ }^{3}$

\title{
(LHCb Collaboration)
}

\author{
${ }^{1}$ Centro Brasileiro de Pesquisas Físicas (CBPF), Rio de Janeiro, Brazil \\ ${ }^{2}$ Universidade Federal do Rio de Janeiro (UFRJ), Rio de Janeiro, Brazil \\ ${ }^{3}$ Center for High Energy Physics, Tsinghua University, Beijing, China \\ ${ }^{4} L A P P$, Université de Savoie, CNRS/IN2P3, Annecy-Le-Vieux, France \\ ${ }^{5}$ Clermont Université, Université Blaise Pascal, CNRS/IN2P3, LPC, Clermont-Ferrand, France \\ ${ }^{6} C P P M$, Aix-Marseille Université, CNRS/IN2P3, Marseille, France \\ ${ }^{7}$ LAL, Université Paris-Sud, CNRS/IN2P3, Orsay, France \\ ${ }^{8}$ LPNHE, Université Pierre et Marie Curie, Université Paris Diderot, CNRS/IN2P3, Paris, France \\ ${ }^{9}$ Fakultät Physik, Technische Universität Dortmund, Dortmund, Germany \\ ${ }^{10}$ Max-Planck-Institut für Kernphysik (MPIK), Heidelberg, Germany \\ ${ }^{11}$ Physikalisches Institut, Ruprecht-Karls-Universität Heidelberg, Heidelberg, Germany \\ ${ }^{12}$ School of Physics, University College Dublin, Dublin, Ireland \\ ${ }^{13}$ Sezione INFN di Bari, Bari, Italy \\ ${ }^{14}$ Sezione INFN di Bologna, Bologna, Italy \\ ${ }^{15}$ Sezione INFN di Cagliari, Cagliari, Italy \\ ${ }^{16}$ Sezione INFN di Ferrara, Ferrara, Italy \\ ${ }^{17}$ Sezione INFN di Firenze, Firenze, Italy \\ ${ }^{18}$ Laboratori Nazionali dell'INFN di Frascati, Frascati, Italy \\ ${ }^{19}$ Sezione INFN di Genova, Genova, Italy \\ ${ }^{20}$ Sezione INFN di Milano Bicocca, Milano, Italy \\ ${ }^{21}$ Sezione INFN di Milano, Milano, Italy \\ ${ }^{22}$ Sezione INFN di Padova, Padova, Italy \\ ${ }^{23}$ Sezione INFN di Pisa, Pisa, Italy \\ ${ }^{24}$ Sezione INFN di Roma Tor Vergata, Roma, Italy \\ ${ }^{25}$ Sezione INFN di Roma La Sapienza, Roma, Italy \\ ${ }^{26}$ Henryk Niewodniczanski Institute of Nuclear Physics Polish Academy of Sciences, Kraków, Poland \\ ${ }^{27}$ AGH-University of Science and Technology, Faculty of Physics and Applied Computer Science, Kraków, Poland \\ ${ }^{28}$ National Center for Nuclear Research (NCBJ), Warsaw, Poland \\ ${ }^{29}$ Horia Hulubei National Institute of Physics and Nuclear Engineering, Bucharest-Magurele, Romania \\ ${ }^{30}$ Petersburg Nuclear Physics Institute (PNPI), Gatchina, Russia \\ ${ }^{31}$ Institute of Theoretical and Experimental Physics (ITEP), Moscow, Russia \\ ${ }^{32}$ Institute of Nuclear Physics, Moscow State University (SINP MSU), Moscow, Russia \\ ${ }^{33}$ Institute for Nuclear Research of the Russian Academy of Sciences (INR RAN), Moscow, Russia \\ ${ }^{34}$ Budker Institute of Nuclear Physics (SB RAS) and Novosibirsk State University, Novosibirsk, Russia \\ ${ }^{35}$ Institute for High Energy Physics (IHEP), Protvino, Russia \\ ${ }^{36}$ Universitat de Barcelona, Barcelona, Spain \\ ${ }^{37}$ Universidad de Santiago de Compostela, Santiago de Compostela, Spain \\ ${ }^{38}$ European Organization for Nuclear Research (CERN), Geneva, Switzerland \\ ${ }^{39}$ Ecole Polytechnique Fédérale de Lausanne (EPFL), Lausanne, Switzerland \\ ${ }^{40}$ Physik-Institut, Universität Zürich, Zürich, Switzerland \\ ${ }^{41}$ Nikhef National Institute for Subatomic Physics, Amsterdam, The Netherlands \\ ${ }^{42}$ Nikhef National Institute for Subatomic Physics and VU University Amsterdam, Amsterdam, The Netherlands \\ ${ }^{43}$ NSC Kharkiv Institute of Physics and Technology (NSC KIPT), Kharkiv, Ukraine \\ ${ }^{44}$ Institute for Nuclear Research of the National Academy of Sciences (KINR), Kyiv, Ukraine \\ ${ }^{45}$ University of Birmingham, Birmingham, United Kingdom \\ ${ }^{46}$ H.H. Wills Physics Laboratory, University of Bristol, Bristol, United Kingdom \\ ${ }^{47}$ Cavendish Laboratory, University of Cambridge, Cambridge, United Kingdom \\ ${ }^{48}$ Department of Physics, University of Warwick, Coventry, United Kingdom
}




\footnotetext{
${ }^{49}$ STFC Rutherford Appleton Laboratory, Didcot, United Kingdom

${ }^{50}$ School of Physics and Astronomy, University of Edinburgh, Edinburgh, United Kingdom

${ }^{51}$ School of Physics and Astronomy, University of Glasgow, Glasgow, United Kingdom

${ }^{52}$ Oliver Lodge Laboratory, University of Liverpool, Liverpool, United Kingdom

${ }^{53}$ Imperial College London, London, United Kingdom

${ }^{54}$ School of Physics and Astronomy, University of Manchester, Manchester, United Kingdom

${ }^{55}$ Department of Physics, University of Oxford, Oxford, United Kingdom

${ }^{56}$ Massachusetts Institute of Technology, Cambridge, MA, United States

${ }^{57}$ University of Cincinnati, Cincinnati, OH, United States

${ }^{58}$ University of Maryland, College Park, MD, United States

${ }^{59}$ Syracuse University, Syracuse, NY, United States

${ }^{60}$ Pontifícia Universidade Católica do Rio de Janeiro (PUC-Rio), Rio de Janeiro, Brazil (associated with Institution Universidade Federal do Rio de Janeiro (UFRJ), Rio de Janeiro, Brazil)

${ }^{61}$ Institute of Particle Physics, Central China Normal University, Wuhan, Hubei, China (associated with Institution Center for High Energy Physics, Tsinghua University, Beijing, China)

${ }^{62}$ Departamento de Fisica, Universidad Nacional de Colombia, Bogota, Colombia (associated with Institution LPNHE, Université Pierre et Marie Curie, Université Paris Diderot, CNRS/IN2P3, Paris, France)

${ }^{63}$ Institut für Physik, Universität Rostock, Rostock, Germany (associated with Institution Physikalisches Institut, Ruprecht-Karls-Universität Heidelberg, Heidelberg, Germany)

${ }^{64}$ National Research Centre Kurchatov Institute, Moscow, Russia (associated with Institution Institute of Theoretical and Experimental Physics (ITEP), Moscow, Russia)

${ }^{65}$ Instituto de Fisica Corpuscular (IFIC), Universitat de Valencia-CSIC, Valencia, Spain (associated with Institution Universitat de Barcelona, Barcelona, Spain

${ }^{66}$ Van Swinderen Institute, University of Groningen, Groningen, The Netherlands (associated with Institution Nikhef National Institute for Subatomic Physics, Amsterdam, The Netherlands)

${ }^{67}$ Celal Bayar University, Manisa, Turkey (associated with Institution European Organization for Nuclear Research (CERN),
} Geneva, Switzerland)

${ }^{a}$ Also at Università di Firenze, Firenze, Italy.

${ }^{\mathrm{b}}$ Also at Università di Ferrara, Ferrara, Italy.

${ }^{c}$ Also at Università della Basilicata, Potenza, Italy.

${ }^{\mathrm{d}}$ Also at Università di Modena e Reggio Emilia, Modena, Italy.

${ }^{\mathrm{e}}$ Also at Università di Milano Bicocca, Milano, Italy.

${ }^{\mathrm{f}}$ Also at LIFAELS, La Salle, Universitat Ramon Llull, Barcelona, Spain.

${ }^{\mathrm{g}}$ Also at Università di Bologna, Bologna, Italy.

${ }^{\mathrm{h}}$ Also at Università di Roma Tor Vergata, Roma, Italy.

${ }^{\mathrm{i}}$ Also at Università di Genova, Genova, Italy.

${ }^{\mathrm{j}}$ Also at Politecnico di Milano, Milano, Italy.

${ }^{\mathrm{k}}$ Also at Universidade Federal do Triângulo Mineiro (UFTM), Uberaba-MG, Brazil.

${ }^{1}$ Also at AGH-University of Science and Technology, Faculty of Computer Science, Electronics and Telecommunications, Kraków, Poland.

${ }^{\mathrm{m}}$ Also at Università di Padova, Padova, Italy.

${ }^{\mathrm{n}}$ Also at Università di Cagliari, Cagliari, Italy.

${ }^{\circ}$ Also at Scuola Normale Superiore, Pisa, Italy.

${ }^{\mathrm{p}}$ Also at Hanoi University of Science, Hanoi, Viet Nam.

${ }^{\mathrm{q}}$ Also at Università di Bari, Bari, Italy.

${ }^{\mathrm{r}}$ Also at Università degli Studi di Milano, Milano, Italy.

${ }^{\text {s}}$ Also at Università di Pisa, Pisa, Italy.

${ }^{\mathrm{t}}$ Also at Università di Roma La Sapienza, Roma, Italy.

uAlso at Università di Urbino, Urbino, Italy.

${ }^{v}$ Also at P.N. Lebedev Physical Institute, Russian Academy of Science (LPI RAS), Moscow, Russia. 